\title{
SUBARU IMAGING OF ASYMMETRIC FEATURES IN A TRANSITIONAL DISK IN UPPER SCORPIUS*
}

\author{
S. Mayama ${ }^{1,2}$, J. Hashimoto ${ }^{3}$, T. Muto ${ }^{4}$, T. Tsukagoshi ${ }^{5}$, N. Kusakabe ${ }^{3}$, M. Kuzuhara $^{3,6}$, Y. TAKahashi $^{3,7}$, T. Kudo $^{8}$, \\ R. Dong ${ }^{9}$, M. Fukagawa ${ }^{10}$, M. TAKami ${ }^{11}$, M. Momose ${ }^{5}$, J. P. Wisniewski ${ }^{12}$, K. Follette ${ }^{13}$, L. Abe $^{14}$, E. AkiYama ${ }^{3}$,

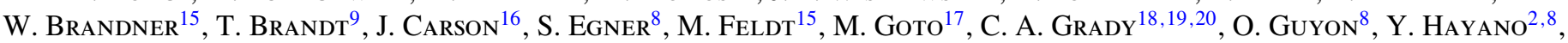

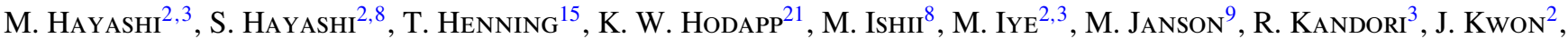

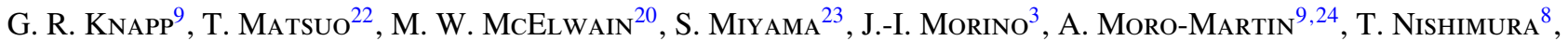

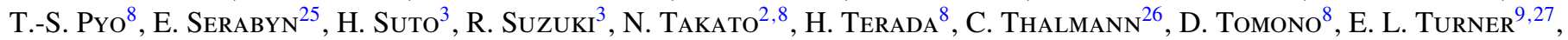 \\ M. Watanabe ${ }^{28}$, T. Yamada ${ }^{29}$, H. TAKAmi ${ }^{2,8}$, T. Usuda ${ }^{2,8}$, and M. TAmura ${ }^{2,3}$ \\ ${ }^{1}$ The Center for the Promotion of Integrated Sciences, The Graduate University for Advanced Studies (SOKENDAI), Shonan International Village, \\ Hayama-cho, Miura-gun, Kanagawa 240-0193, Japan; mayama_satoshi@soken. ac.jp \\ ${ }^{2}$ Department of Astronomical Science, The Graduate University for Advanced Studies (SOKENDAI), 2-21-1 Osawa, Mitaka, Tokyo 181-8588, Japan \\ ${ }^{3}$ National Astronomical Observatory of Japan, 2-21-1 Osawa, Mitaka, Tokyo 181-8588, Japan \\ ${ }^{4}$ Division of Liberal Arts, Kogakuin University, 1-24-2, Nishi-Shinjuku, Shinjuku-ku, Tokyo 163-8677, Japan \\ ${ }^{5}$ College of Science, Ibaraki University, 2-1-1 Bunkyo, Mito, Ibaraki 310-8512, Japan \\ ${ }^{6}$ Department of Earth and Planetary Science, The University of Tokyo, Hongo 7-3-1, Bunkyo-ku, Tokyo 113-0033, Japan \\ ${ }^{7}$ Department of Astronomy, The University of Tokyo, Hongo 7-3-1, Bunkyo-ku, Tokyo 113-0033, Japan \\ ${ }^{8}$ Subaru Telescope, 650 North A'ohoku Place, Hilo, HI 96720, USA \\ ${ }^{9}$ Department of Astrophysical Sciences, Princeton University, NJ 08544, USA \\ ${ }^{10}$ Department of Earth and Space Science, Graduate School of Science, Osaka University, 1-1 Machikaneyama, Toyonaka, Osaka 560-0043, Japan \\ ${ }^{11}$ Institute of Astronomy and Astrophysics, Academia Sinica, P.O. Box 23-141, Taipei 106, Taiwan \\ ${ }^{12}$ H L Dodge Department of Physics and Astronomy, University of Oklahoma, 440 West Brooks St. Norman, OK 73019, USA \\ ${ }^{13}$ Department of Astronomy and Steward Observatory, The University of Arizona, 933 North Cherry Avenue, Rm. N204, Tucson, AZ 85721-0065, USA \\ ${ }^{14}$ Laboratoire Hippolyte Fizeau, UMR6525, Universite de Nice Sophia-Antipolis, 28, avenue Valrose, F-06108 Nice Cedex 02, France \\ ${ }^{15}$ Max Planck Institute for Astronomy, Koenigstuhl 17, D-69117 Heidelberg, Germany \\ ${ }^{16}$ Department of Physics and Astronomy, College of Charleston, 58 Coming Street, Charleston, SC 29424, USA \\ 17 Universitäts-Sternwarte München Scheinerstr. 1, D-81679, Munich, Germany \\ ${ }^{18}$ Goddard Center for Astrobiology, NASA's Goddard Space Flight Center, Greenbelt, MD 20771, USA \\ ${ }^{19}$ Eureka Scientific, 2452 Delmer, Suite 100, Oakland, CA 96002, USA \\ ${ }^{20}$ ExoPlanets and Stellar Astrophysics Laboratory, Code 667, Goddard Space Flight Center, Greenbelt, MD 20771, USA \\ ${ }^{21}$ Institute for Astronomy, University of Hawaii, 640 North A'ohoku Place, Hilo, HI 96720, USA \\ ${ }^{22}$ Department of Astronomy, Kyoto University, Kitashirakawa-Oiwake-cho, Sakyo-ku, Kyoto 606-8502, Japan \\ ${ }^{23}$ Office of the President, Hiroshima University, 1-3-2 Kagamiyama, Higashi-Hiroshima, Hiroshima 739-8511, Japan \\ ${ }^{24}$ Departamento de Astrofisica, CAB (INTA-CSIC), Instituto Nacional de Técnica Aeroespacial, Torrejón de Ardoz, E-28850 Madrid, Spain \\ ${ }^{25}$ Jet Propulsion Laboratory, California Institute of Technology, Pasadena, CA 91109, USA \\ ${ }^{26}$ Astronomical Institute "Anton Pannekoek," University of Amsterdam, Science Park 904, 1098 XH Amsterdam, The Netherlands \\ ${ }^{27}$ Institute for the Physics and Mathematics of the Universe, The University of Tokyo, Kashiwa 227-8568, Japan \\ ${ }^{28}$ Department of Cosmosciences, Hokkaido University, Sapporo 060-0810, Japan \\ ${ }^{29}$ Astronomical Institute, Tohoku University, Aoba, Sendai 980-8578, Japan \\ Received 2012 August 11; accepted 2012 October 1; published 2012 November 7
}

\begin{abstract}
We report high-resolution $(0.07$ arcsec) near-infrared polarized intensity images of the circumstellar disk around the star 2MASS J16042165-2130284 obtained with HiCIAO mounted on the Subaru $8.2 \mathrm{~m}$ telescope. We present our $H$-band data, which clearly exhibit a resolved, face-on disk with a large inner hole for the first time at infrared wavelengths. We detect the centrosymmetric polarization pattern in the circumstellar material as has been observed in other disks. Elliptical fitting gives the semimajor axis, semiminor axis, and position angle (P.A.) of the disk as $63 \mathrm{AU}, 62 \mathrm{AU}$, and $-14^{\circ}$, respectively. The disk is asymmetric, with one dip located at P.A.s of $\sim 85^{\circ}$. Our observed disk size agrees well with a previous study of dust and $\mathrm{CO}$ emission at submillimeter wavelength with Submillimeter Array. Hence, the near-infrared light is interpreted as scattered light reflected from the inner edge of the disk. Our observations also detect an elongated arc (50 AU) extending over the disk inner hole. It emanates at the inner edge of the western side of the disk, extending inward first, then curving to the northeast. We discuss the possibility that the inner hole, the dip, and the arc that we have observed may be related to the existence of unseen bodies within the disk.
\end{abstract}

Key words: planetary systems - protoplanetary disks - stars: pre-main sequence - techniques: polarimetric

\section{INTRODUCTION}

Planets are believed to form in protoplanetary disks. To study the structures of protoplanetary disks, multi-wavelength observations have targeted such disks around young stellar objects located in nearby star-forming regions such as the

\footnotetext{
* Based on data collected at the Subaru Telescope, which is operated by the National Astronomical Observatory of Japan.
}

Taurus, $\rho$ Oph, and Upper Scorpius regions (e.g., Kenyon \& Hartmann 1995; Preibisch \& Zinnecker 1999; Furlan et al. 2006; Andrews et al. 2009; Dahm \& Carpenter 2009; McClure 2009; Andrews et al. 2011; Mathews et al. 2012a). Among such nearby star-forming regions, Upper Scorpius, which is located at a distance of $145 \mathrm{pc}$ (de Zeeuw et al. 1999), provides a unique environment, particularly in terms of its age. While the age is only 5-10 Myr (Pecaut et al. 2012; Song et al. 2012), the lack of dense molecular material and embedded young stellar objects 
indicate that the star formation process in Upper Scorpius is over. The area is essentially free of dense gas and dust clouds, and the association members show only moderate extinctions $\left(A_{\mathrm{V}}<2 \mathrm{mag}\right)$. In fact, there are only two $K$ and $M$ stars $(1.5 \%)$, including the star discussed here, in the Upper Scorpius region which exhibit a $K$-band excess, although about half of the stars in Taurus exhibit a $K$-band excess (Strom et al. 1989). This implies that there are very few stars still hosting protoplanetary disks in Upper Scorpius star-forming region.

Here, we introduce our target 2MASS J16042165-2130284 (hereafter J1604-2130) in Upper Scorpius. J1604-2130 was first identified as a member of the Upper Scorpius OB association in the spectroscopic survey of X-ray-selected sources of Preibisch \& Zinnecker (1999), who found: stellar age 3.7 Myr, spectral type $\mathrm{K} 2$, stellar mass $1 M_{\odot}, \log T_{\text {eff }}[\mathrm{K}]=3.658$, and $\log \left[L / L_{\odot}\right]=-0.118$. The spectral energy distribution (SED) of J1604-2130 has the features of a transitional disk (Dahm \& Carpenter 2009), in which small dust grains have been partly cleared from the inner disk but are still abundant at larger radii. Recently, Mathews et al. (2012b) presented the Submillimeter Array (SMA) $880 \mu \mathrm{m}$ images, which directly resolved a faceon protoplanetary disk around J1604-2130, and derived a dust mass of $0.1 M_{\text {Jup }}$. The availability of radio data was one advantage to selecting this target; however, other advantages abound and are as follows: (1) J1604-2130 is located in a nearby starforming region, Upper Sco; (2) it hosts one of the largest inner holes in transitional disks discovered to date; (3) it hosts the most massive disk in Upper Scorpius; (4) the inclination $6^{\circ} \pm 1.5$ (Mathews et al. 2012b) is very close to face-on.

Infrared photometry and spectroscopy with Spitzer has shown that less than $\sim 10 \%$ of stars with disks show some degree of inner disk depletion yet still retain massive outer disks (e.g., Cieza et al. 2010; Muzerolle et al. 2010; Luhman et al. 2011). Although there have been several transitional disks detected with radio observations with SMA around single young solar mass sources (e.g., Piétu et al. 2006; Andrews et al. 2011), only a handful of transitional disks have been detected in the near-infrared (e.g., Fukagawa et al. 2006; Thalmann et al. 2010; Hashimoto et al. 2011). This is mainly because it is difficult to resolve structures with only a few 10 AU scale close to a bright central star. Furthermore, structures inside the hole of disks have rarely been directly imaged or resolved to date. To gain a better understanding of the morphological structures at radii where planets are expected to form, we conducted high-resolution near-infrared polarimetric observations of J1604-2130.

\section{OBSERVATIONS AND DATA REDUCTION}

We carried out polarimetry in $H$ band $(1.6 \mu \mathrm{m})$ toward J1604-2130 using the high-resolution imaging instrument HiCIAO (Tamura et al. 2006; Hodapp et al. 2006) with a dual-beam polarimeter at the Subaru $8.2 \mathrm{~m}$ Telescope on 2012 April 11. These observations are part of the ongoing highcontrast imaging survey (SEEDS; Tamura 2009). The polarimetric observation mode acquires $o$-rays and $e$-rays simultaneously, and images a field of view of $10^{\prime \prime} \times 20^{\prime \prime}$ with a pixel scale of 9.53 mas pixel $^{-1}$. J1604-2130 was observed without an occulting mask in order to image the inner most region around the central star. The exposures were performed at four position angles (P.A.s) of the half-wave plate, with a sequence of P.A. $=0^{\circ}$, $45^{\circ}, 22.5$, and 67.5 to measure the Stokes parameters. The integration time per wave plate position was $15 \mathrm{~s}$ and we obtained 25 wave plate cycles. The adaptive optics system (AO188; Hayano et al. 2010) provides a diffraction limited and mostly stable stel-
Table 1

The Result of the Ellipse Fitting for the Disk of J1604-2130

\begin{tabular}{lc}
\hline \hline Semimajor axis & $63.4 \pm 1.0(\mathrm{AU})$ \\
Semiminor axis & $62.4 \pm 1.0(\mathrm{AU})$ \\
Ellipse center $(u, v)^{\mathrm{a}}$ & $(-1.1 \pm 1.1,1.5 \pm 1.1)($ pixel) \\
Position angle & $-14^{\circ} \pm 11^{\circ}$ \\
Inclination & $10^{\circ} 2 \pm 10^{\circ} 1$ \\
\hline
\end{tabular}

Note. ${ }^{a}$ Central position $(0,0)$ corresponds to the stellar position.

lar point spread function (PSF). The total integration time of the polarization intensity (hereafter $P I$ ) image was $750 \mathrm{~s}$ after removing low-quality images with large FWHMs by careful inspections of the stellar PSF.

The Image Reduction and Analysis Facility (IRAF ${ }^{30}$ ) software was used for all data reduction. The polarimetric data reduction technique is described by Hashimoto et al. (2011) and Muto et al. (2012), using the standard approach for polarimetric differential imaging (Hinkley et al. 2009). By subtracting two images of extraordinary and ordinary rays at each wave plate position, we obtained $+Q,-Q,+U$, and $-U$ images, from which $2 Q$ and $2 U$ images were made by another subtraction to eliminate remaining aberration. The $P I$ was then calculated using $P I=\sqrt{Q^{2}+U^{2}}$. The instrumental polarization of HiCIAO at the Nasmyth platform was corrected for by following Joos et al. (2008).

\section{RESULTS}

The $H$-band $P I$ image of J1604-2130 is presented in Figure 1(a). The emission arises from dust particles mixed with gas in the circumstellar structures, scattering the stellar light. Details of the near-infrared disk around J1604-2130

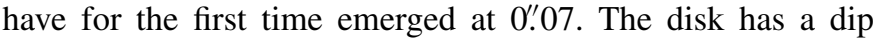
D, located around a P.A. $\sim 85^{\circ}$, making this transitional disk asymmetric. Inside the bright disk, we see a region where the surface brightness drops by a factor of $\sim 5$ compared with the disk. We call this inner lower-brightness region the "hole" throughout the remainder of this paper. Figure 1(b) shows $H$-band polarization vectors. The disk with its centrosymmetric polarization pattern surrounds the central source J1604-2130 as previously observed in other disks using the technique of nearinfrared imaging polarimetry (e.g., Hashimoto et al. 2011).

Elliptical fitting was performed to measure several disk parameters including the angular separations of the major and minor axes, the P.A., inclination, and the position of the ellipse center. We first measured disk radial profiles in $5^{\circ} \mathrm{P}$. A. increments, and then extracted coordinates of the brightest peak area. Those coordinates were used to fit an ellipse. The nonlinear least-squares Levenburg-Marquardt algorithm with the above five free parameters was performed and its result is displayed in Table 1. Its shape is superimposed on Figure 1(a). The derived semimajor and semiminor axes are called "NIR peak radii" in this paper. The geometric center of the disk is consistent with the position of the star within errors. The disk P. A. has a relatively large error because the disk is close to circular.

Figure 2 shows the azimuthal surface brightness at $r=33$, 63, $145 \mathrm{AU}$, which are illustrated in Figure 1(c), with the P. A. measured from north to east. The error bars shown in Figure 2 represent the calculated standard deviation. The left

\footnotetext{
30 IRAF is distributed by National Optical Astronomy Observatory, which is operated by the Association of Universities for Research in Astronomy, Inc., under cooperative agreement with the National Science Foundation.
} 

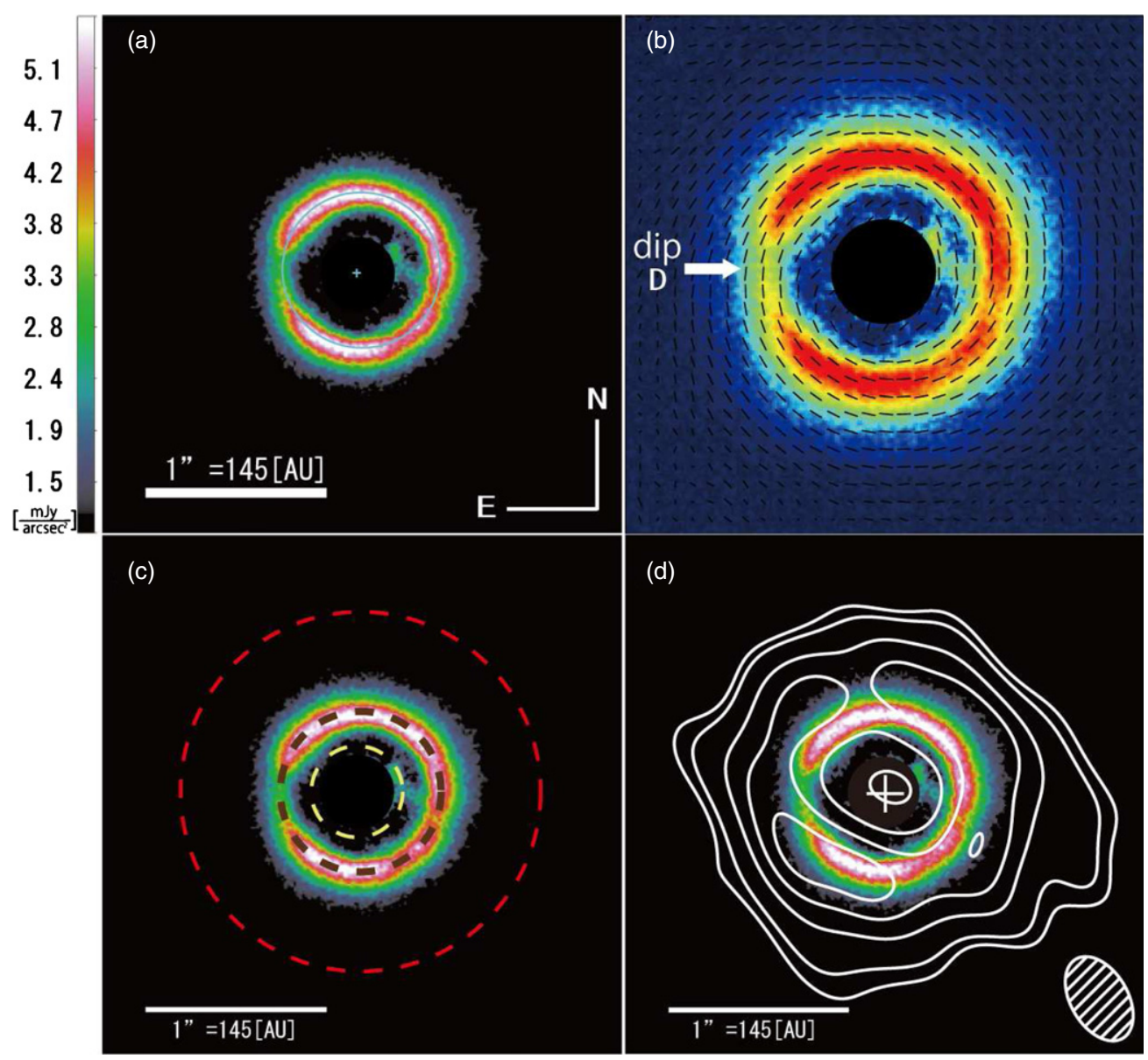

Figure 1. $H$-band HiCIAO images of J1604-2130. The saturated central area (radius $=0 ! 2$ ) is masked in black. (a) The $P I$ image of J1604-2130. The field of view

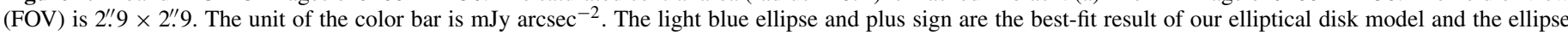
center. (b) $H$-band polarization vectors superposed on the $P I$ image. The vector directions indicate angles of polarization. The plotted vectors are based on 7 [pixel] $\times$ 7 [pixel] binning corresponding to the spatial resolution. The FOV is 2 ". $0 \times 22^{\prime \prime} .0$. The vector's lengths are arbitrary. (c) Red ( $r=145$ AU), brown ( $r=63$ AU), and yellow ( $r=33 \mathrm{AU}$ ) circles, corresponding Figure 2, superimposed on the PI image. (d) SMA $880 \mu \mathrm{m}$ continuum map (Mathews et al. 2012b) superimposed on the $P I$ image. White color contours indicate $2 \sigma, 3 \sigma, 6 \sigma, 9 \sigma$, and $12 \sigma$ intensity $\left(1 \sigma=1.3 \mathrm{mJy} \mathrm{beam}^{-1}\right)$. The $\sim 0^{\prime \prime} .5 \times 00^{\prime \prime} 3$ beam of SMA is shown in the bottom right.

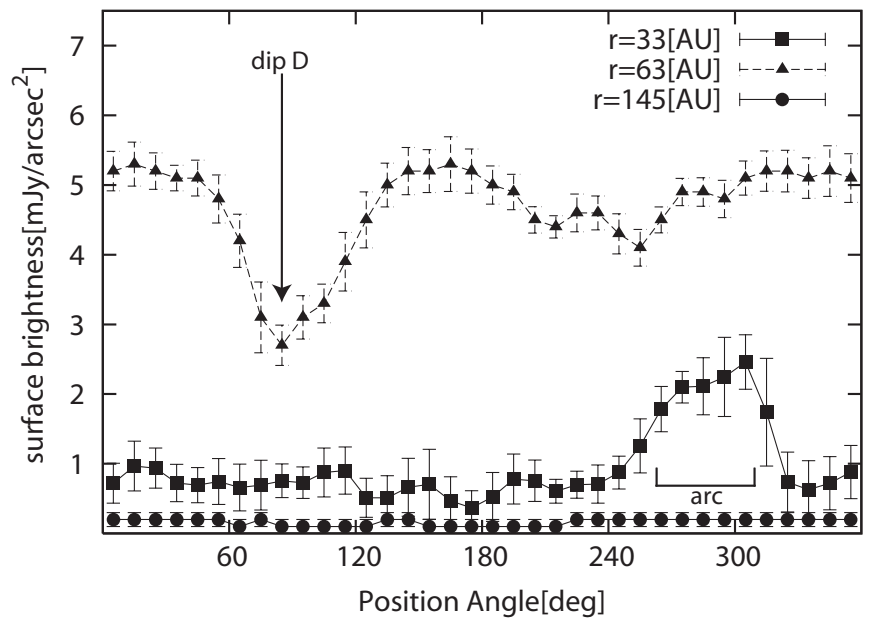

Figure 2. Azimuthal surface brightness profile at $r=33 \mathrm{AU}$ (inner hole region), $r=63 \mathrm{AU}$ (inner disk region), and $r=145 \mathrm{AU}$ (outer region) with position angle measured from north to east. The radius $33 \mathrm{AU}$ is measured as it is the brightest part of the arc.

panel of Figure 3 shows the radial surface brightness profile of J1604-2130 along the minor axis. The error bars shown in Figure 3 represent the calculated standard deviation. In the north, the surface brightness along the minor axis increases as $r^{3.6 \pm 0.2}$ from 40 to $62 \mathrm{AU}$, and decreases as $r^{-4.7 \pm 0.1}$ from 74 to $124 \mathrm{AU}$. In the south, the surface brightness along the minor axis increases as $r^{3.0 \pm 0.4}$ from 40 to $62 \mathrm{AU}$, and decreases as $r^{-4.7 \pm 0.2}$ from 74 to $124 \mathrm{AU}$. The right panel of Figure 3 shows the radial profile of the surface brightness along the major axis. In the east, the surface brightness along the major axis increases as $r^{2.6 \pm 0.2}$ from 40 to $62 \mathrm{AU}$, and decreases as $r^{-4.7 \pm 0.1}$ from 74 to $124 \mathrm{AU}$. In the west, the surface brightness along the major axis increases as $r^{1.9 \pm 0.2}$ from 40 to $62 \mathrm{AU}$, and decreases as $r^{-4.0 \pm 0.2}$ from 74 to $124 \mathrm{AU}$. The profiles along both the major and minor axes show a change of slope beyond $63 \mathrm{AU}$, thus reconfirming the NIR peak radius to be 63 AU. Along all four axes, the counts drop to half of the peak counts at around $50 \mathrm{AU}$ from the central star. The width of the brightest region where counts exceeds half of the peak counts in the disk is around 30 AU.

A salient feature inside the hole is an elongated arc extending over the disk inner hole. It begins at the inner edge of the western side of the disk, extending inward first, then curving to the northeast. The whole arc extends at least $50 \mathrm{AU}$. This arc is equivalent in brightness to the inner edge of the disk and to the dip $\mathrm{D}$ region. This arc consists of a real feature and is not an artifact because in contrast to the inner hole errors (shown as squares in Figure 2), the surface brightness difference between the arc and the other regions in the inner hole has a $6 \sigma$ significance, whereas the other directions show 


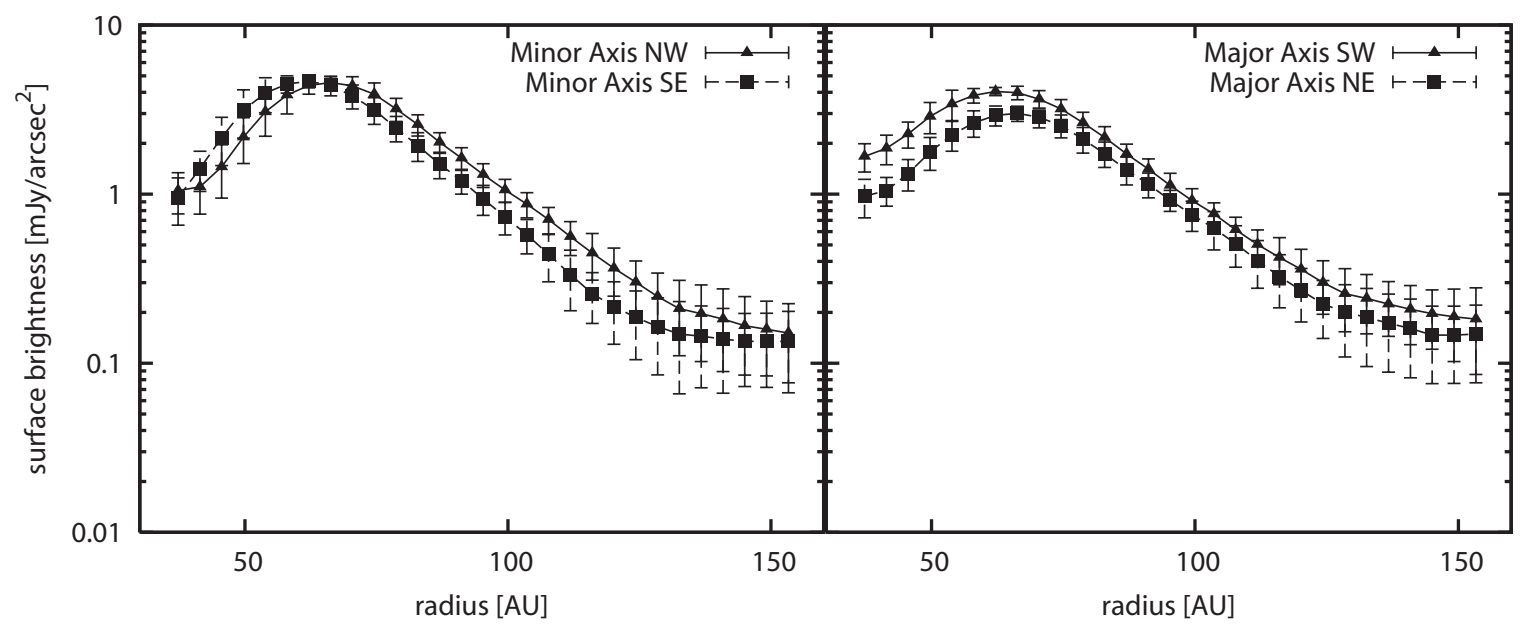

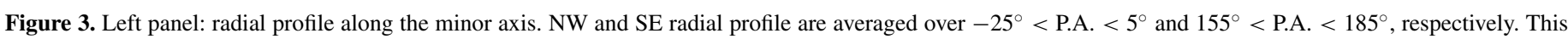

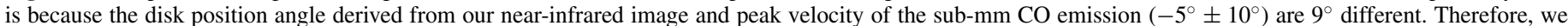

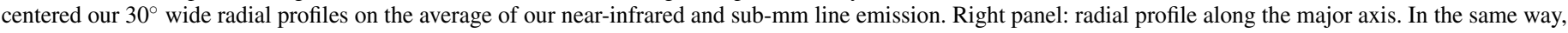
$\mathrm{NE}$ and SW radial profiles are averaged over $65^{\circ}<$ P.A. $<95^{\circ}$ and $245^{\circ}<$ P.A. $<275^{\circ}$, respectively.

small fluctuations in emission. Furthermore, the polarization vectors in the region of the arc mostly face the central star as well as the region of the bright disk, indicating that the arc is also illuminated by central star and is physically connected to inner edge of the disk.

In the same way, the dip D is a real dip feature and is not an artifact because the difference in surface brightness between $\mathrm{D}$ and the rest of the disk is statistically significant and not within error bars. In addition, the polarization vectors in the region of the dip mostly face the central star.

There appears to be another dip located around a P. A. $\sim 255^{\circ}$, which is not azimuthally symmetric with $\mathrm{D}$ in terms of location and surface brightness. However, this another dip is not significant outside of the uncertainties of the observations, thus follow-up future observations are required to confirm its presence.

\section{DISCUSSION}

\subsection{Comparison with the SMA Imaging}

Mathews et al. (2012b) present SMA $880 \mu \mathrm{m}$ continuum and CO (3-2) line observations of this source with a resolution of 0 .'34 and resolve the disk (Figure 1(d)). They determine the surface density distribution of the disk with inner cavity whose radius is $72 \mathrm{AU}$ based on the model fitting to the visibility at $880 \mu \mathrm{m}$. They also suggest that sparse matter still remains inside the cavity (20-72 AU) because of the dust emission indicated by mid-infrared (Carpenter et al. 2009) excesses and their model fitting. The NIR peak disk radius of 63 AU derived from our $H$-band image roughly agrees or may be slightly smaller than their measurement and can be explained by scattered light reflected at the inner edge of the disk.

Based on their CO peak velocity map, Mathews et al. (2012b) derive the P.A.s of the major and minor axes to be $85^{\circ} \pm 10^{\circ}$ and $-5^{\circ} \pm 10^{\circ}$, respectively. Their derive disk parameters, including the P.A. and inclination, are in a good agreement with values derived from our near infrared observations.

Mathews et al. (2012b) point out that the relatively high mass of the gas-dust disk, its sharp inner edge, and the lack of gas accretion onto the star together point to a dynamical origin for the inner hole. No companions have been found down to brown dwarf masses (Kraus et al. 2008; Ireland et al. 2011). Based on their analysis, they suggest that the origin of the hole can be attributed to the unseen presence of one or several giant planets within about a $40 \mathrm{AU}$.

The width and depth of the hole depend on the planet mass, disk viscosity, and disk thickness (e.g., Takeuchi et al. 1996; Crida et al. 2006). As discussed by Dong et al. (2012a, 2012b), the radius of the inner hole is on the order of $\sim 60-70 \mathrm{AU}$. There have been extensive studies on the process of gap opening by a planet embedded in a disk (e.g., Lin \& Papaloizou 1986; Takeuchi et al. 1996; Crida et al. 2006). A rough estimate of the upper limit of the size of the gap that one planet can make may be given by the location of the lowest order Lindblad resonances, which, in the case of the outer resonance (the outer edge of the gap), is at $\sim 1.6$ times the orbital radius of the embedded planet (Takeuchi et al. 1996). If this is the case, the existence of the inner hole might indicate the existence of at least one planet at 40-50 AU from the central star (see also Crida et al. 2006), and the inner edge of the hole would be expected at 25-30 AU, which is inside of our saturation radius and is therefore unresolved in this study.

If multiple planets reside within the hole, the hole width can be wider and the inner disk may be much smaller than the single planet case (Zhu et al. 2011). To understand the properties of the whole of this object, it is important to give constraints on the inner disk structure using detailed SED modeling (see Dong et al. 2012a, 2012b) and/or high-resolution observations by ALMA. Our observations do not show a clear inner disk edge, suggesting that the inner disk radius must be inside of the saturation radius of our observations.

\subsection{Non-axisymmetric Features}

We list a few possibilities for origin of the two nonaxisymmetric features, the dip and the arc since it is difficult to draw firm conclusions at this stage. According to Figure 6(c) in Mathews et al. (2012b), the only structures that are not reproduced by the axisymmetric disk model within $2 \sigma$ error are the southeast component; the north dip is consistent with the axisymmetric disk model convolved with the elliptical beam. We, therefore, do not elaborate on the north dip on the submillimeter (sub-mm) disk in the following discussions. Mathews et al. (2012b) observe that the millimeter continuum emission has a peak at 0.5 southeast from the star in the $880 \mu \mathrm{m}$ image. 
A similar distribution is also seen in the total integrated intensity distribution of $\mathrm{CO}(3-2)$ emission. Our $H$-band image and Figure 3 show that the southeast of the disk is brighter than the east and the west, despite the fact that we observe the scattered light originating at the disk surface. This may be explained in a way that the more materials exist, the thicker the disk is, resulting in a higher grazing angle of the stellar light at the scattering surface (e.g., Muto 2011). In our near-infrared image, the north of the disk is also bright. The brightness of the disk in the north, together with sub-mm observations suggesting material accumulation in the south, infers that the northern portion of the disk is the near side. This assumes that forward scattering dominates. If this picture of the disk orientation is correct, the disk rotation is counterclockwise direction. This geometry might also explain the origins of the non-axisymmetric structures at $\mathbf{D}$. $\mathbf{D}$ is at the interface between where the material accumulated (south) and where forward scattering is significant (north). However, it should be noted that since the disk is nearly face-on, significant forward scattering excess and precise prescriptions of the phase function should be required, and our discussion crucially depends on the details of the optical properties of the dust particles. It should also be noted that the material concentration in the south is within $2 \sigma$ of the current sub-mm data (Mathews et al. 2012b). The detection of the non-axisymmetric structures at sub-mm wavelengths is needed to examine the details of the disk geometry.

Another possibility for the origin of the $\operatorname{dip} \mathbf{D}$ is that there is a massive gravitating object at the disk's midplane. It could be a planet (which is not necessarily the same as the one discussed in the previous section as the origin of the inner hole), a clump formed by hydrodynamic instability (e.g., gravitational instability), or something else. Such objects can pull the materials from the surface toward the disk's midplane, thereby producing a shadow in the scattered light image (Jang-Condell 2009). This might produce features such as D. Such objects have sizes smaller than the SMA beam size, therefore cannot be resolved with the SMA.

For the arc structure in the west, the morphology is similar to spiral density waves in the disk. An arguably uniqueness is that this arc extends over the "disk inner hole" region. It also resembles to spiral density waves in terms of the pitch angle. Spiral waves can be produced by dynamical processes in the disk such as the turbulence or the planet-disk interaction (see, e.g., Papaloizou et al. 2007). If this feature is a spiral wave, its morphology indicates that the disk rotation is counterclockwise because spirals are in general trailing features. This rotation direction is consistent with the picture presented at the beginning of this section. Last, we note that the arc structure shows morphological similarity to the "planet shadows" discussed by Jang-Condell (2009). One possible way to distinguish the different scenarios is to observe the structures of the disk's midplane at longer wavelength. For instance, if a density enhancement is observed at the location of $\mathbf{D}$, this might indicate the existence of a massive body there. If we see an arc structure in the sub-mm as well, then it is likely a density wave.

Another possible way to reveal the nature of the arc may be the contemporaneous SED and scattered light observations. Previous studies indicate that substantial variability has also been seen in MIR SED and has been linked to variable structures including arcs or rims of the disk (Dahm \& Carpenter 2009; Wisniewski et al. 2008; Muzerolle et al. 2009; Espaillat et al. 2011; Flaherty et al. 2012). For the J1604-2130 case, the location of the arc and the rim of the disk wall is generally consistent with the radial location in the disk which possibly contributes to the SED variability.

We thank the telescope staff and operators at the Subaru Telescope for their assistance. We also thank our referee for the constructive comments that have helped to improve this manuscript. Part of this research was carried out at the JPL, under a contract with NASA. This work is partially supported by a Grant-in-Aid for Science Research in a Priority Area from MEXT 22000005 (M.T.), KAKENHI 23103004 (M.M. and M.F.), 24103504 (T.T.), 24840037 (T.M.), US NSF grant 1009314 (J.P.W.), and 1009203 (J.C.). This work was supported in part by the Center for the Promotion of Integrated Sciences (CPIS) of The Graduate University for Advanced Studies (SOKENDAI).

\section{REFERENCES}

Andrews, S. M., Wilner, D. J., Espaillat, C., et al. 2011, ApJ, 732, 42

Andrews, S. M., Wilner, D. J., Hughes, A. M., Qi, C., \& Dullemond, C. P. 2009, ApJ, 700, 1502

Carpenter, J. M., Mamajek, E. E., Hillenbrand, L. A., \& Meyer, M. R. 2009, ApJ, 705,1646

Cieza, L. A., Schreiber, M. R., Romero, G. A., et al. 2010, ApJ, 712, 925

Crida, A., Morbidelli, A., \& Masset, F. 2006, Icarus, 181, 587

Dahm, S. E., \& Carpenter, J. M. 2009, AJ, 137, 4024

de Zeeuw, P. T., Hoogerwerf, R., de Bruijne, J. H. J., Brown, A. G. A., \& Blaauw, A. 1999, AJ, 117, 354

Dong, R., Rafikov, R., Hashimoto, J., et al. 2012b, ApJ, submitted

Dong, R., Rafikov, R., Zhu, Z., et al. 2012a, ApJ, 750, 161

Espaillat, C., Furlan, E., D’Alessio, P., et al. 2011, ApJ, 728, 49

Flaherty, K. M., Muzerolle, J., Rieke, G., et al. 2012, ApJ, 748, 71

Fukagawa, M., Tamura, M., Itoh, Y., et al. 2006, ApJ, 636, L153

Furlan, E., Hartmann, L., Calvet, N., et al. 2006, ApJS, 165, 568

Hashimoto, J., Tamura, M., Muto, T., et al. 2011, ApJ, 729, L17

Hayano, Y., Takami, H., Oya, S., et al. 2010, Proc. SPIE, 7736, 21

Hinkley, S., Oppenheimer, B. R., Soummer, R., et al. 2009, ApJ, 701, 804

Hodapp, K. W., Tamura, M., Suzuki, R., et al. 2006, Proc. SPIE, 6269, 62693

Ireland, M. J., Kraus, A., Martinache, F., Law, N., \& Hillenbrand, L. A. 2011, ApJ, 726, 113

Jang-Condell, H. 2009, ApJ, 700, 820

Joos, F., Buenzli, E., Schmid, H. M., \& Thalmann, C. 2008, Proc. SPIE, 7016, 48

Kenyon, S. J., \& Hartmann, L. 1995, ApJS, 101, 117

Kraus, A. L., Ireland, M. J., Martinache, F., \& Lloyd, J. P. 2008, ApJ, 679, 762

Lin, D. N. C., \& Papaloizou, J. 1986, ApJ, 307, 395

Luhman, K. L., Burgasser, A. J., \& Bochanski, J. J. 2011, ApJ, 730, L9

Mathews, G. S., Williams, J. P., Ménard, F., et al. 2012a, ApJ, 745, 23

Mathews, G. S., Williams, J. P., \& Ménard, F. 2012b, ApJ, 753, 59

McClure, M. 2009, ApJ, 693, L81

Muto, T. 2011, ApJ, 739, 10

Muto, T., Grady, C. A., Hashimoto, J., et al. 2012, ApJ, 748, L22

Muzerolle, J., Allen, L. E., Megeath, S. T., Hernández, J., \& Gutermuth, R. A. 2010, ApJ, 708, 1107

Muzerolle, J., Flaherty, K., Balog, Z., et al. 2009, ApJ, 704, L15

Song, I., Zuckerman, B., \& Bessell, M. S. 2012, AJ, 144, 8

Papaloizou, J. C. B., Nelson, R. P., Kley, W., Masset, F. S., \& Artymowicz, P. 2007, in Protostars and Planets V, ed. B. Reipurth, D. Jewitt, \& K. Keil (Tucson, AZ: Univ. Arizona Press), 655

Pecaut, M. J., Mamajek, E. E., \& Bubar, E. J. 2012, ApJ, 746, 154

Piétu, V., Dutrey, A., Guilloteau, S., Chapillon, E., \& Pety, J. 2006, A\&A, 460, L43

Preibisch, T., \& Zinnecker, H. 1999, AJ, 117, 2381

Strom, K. M., Strom, S. E., Edwards, S., Cabrit, S., \& Skrutskie, M. F. 1989, AJ, 97,1451

Takeuchi, T., Miyama, S. M., \& Lin, D. N. C. 1996, ApJ, 460, 832

Tamura, M. 2009, in AIP Conf. Ser. 1158, Exoplanets and Disks: Their Formation and Diversity, ed. T. Usuda, M. Ishii, \& M. Tamura (Melville, NY: AIP), 11

Tamura, M., Hodapp, K., Takami, H., et al. 2006, Proc. SPIE, 6269, 28

Thalmann, C., Grady, C. A., Goto, M., et al. 2010, ApJ, 718, L87

Wisniewski, J. P., Clampin, M., Grady, C. A., et al. 2008, ApJ, 682, 548

Zhu, Z., Nelson, R. P., Hartmann, L., Espaillat, C., \& Calvet, N. 2011, ApJ, 729,47 